\title{
UNA OBRA INÉDITA DEL PINTOR PUERTORRIQUEÑO FRANCISCO OLLER: ANEURISMA DE LA AORTA, 1867-1871
}

\author{
José G. Rigau PÉREZ 1 \\ Academia Puertorriqueña de la Historia
}

\begin{abstract}
Se presenta el diagrama a color de un aneurisma de la aorta, obra inédita del pintor puertorriqueño Francisco Oller. La obra ilustra un informe clínico manuscrito que el médico francés Henri Dumont redactó entre 1867 y 1871 en Puerto Rico y envió a la Real Academia de Medicina de Madrid. Ambos autores, el pintor entrenado en Madrid y París y el médico investigador en áreas tropicales, fundan su obra en el estudio de condiciones locales, en diálogo con redes internacionales de su profesión.

Palabras clave: Francisco Oller Cestero; Henri Joseph (Enrique José) Dumont; Real Academia de Medicina (Madrid); Puerto Rico; ilustración médica; aneurisma de la aorta.
\end{abstract}

\section{AN UNKNOWN WORK BY THE PUERTORICAN PAINTER FRANCISCO OLLER:}

\section{AORTIC ANEURISM, 1867-1871}

The author presents the color diagram of an aortic aneurism, a previously unknown work by the Puerto Rican painter Francisco Oller. It illustrates a manuscript clinical report by the French physician Henri Dumont, prepared in Puerto Rico between 1867 and 1871 and sent to the Royal Academy of Medicine in Madrid. Both authors, the painter trained in Madrid and Paris and the physician-researcher in tropical areas, base their work on the study of local conditions, in dialogue with international professional networks.

Key words: Francisco Oller Cestero; Henri Joseph (Enrique José) Dumont; Real Academia de Medicina (Madrid); Puerto Rico; medical illustration; aortic aneurism.

Cómo citar este artículo / Citation: Rigau Pérez, José G. (2019): "Una obra inédita del pintor puertorriqueño Francisco Oller: aneurisma de la aorta, 1867-1871”. En: Archivo Español de Arte, vol. 92, núm. 367, Madrid, pp. 293-300. https://doi.org/10.3989/aearte.2019.19.

El pintor Francisco Oller es recordado por presentar, magistralmente, la belleza natural de la Isla y denunciar las crueldades de una sociedad que toleraba la esclavitud y la superstición. La memoria del médico francés Henri Joseph Dumont ha persistido en Puerto Rico por sus publicaciones científicas, sus actividades filantrópicas y el establecimiento de una familia de larga descendencia. El hallazgo de una ilustración anatómica fruto de la colaboración de estos dos personajes motiva este artículo.

La obra añade un eslabón a una cadena de ilustraciones médicas puertorriqueñas que se remonta a finales del siglo XVIII. Entonces se enviaron a Madrid un retrato y un modelo anatómico de niños con malformaciones congénitas, para consultar a los profesores del Real Colegio de Cirugía. Lamentablemente, las obras ya no aparecen. En 1808, José Campeche (1751-1809, el

\footnotetext{
1 jos.rigau@gmail.com / ORCID iD: http://orcid.org/0000-0002-7952-8646.
} 
gran pintor de Puerto Rico antes de Oller) retrató un infante con malformaciones, a instancias del obispo. Quizás la invasión francesa frustró la intención de enviar el cuadro a Madrid y por eso se conserva en San Juan. Agustín Stahl, médico contemporáneo de Oller, marcó otro hito de la gráfica científica con sus cientos de acuarelas botánicas ${ }^{2}$. Las investigaciones locales permitieron a Stahl, criado en Puerto Rico, educado en Alemania, insertarse en una red científica internacional. Oller, por sus estudios juveniles y visitas posteriores a París, aun pintando en la Isla, formó parte de una red artística de vanguardia. Dumont, en posición inversa a las de Oller y Stahl, proyectó la realidad puertorriqueña al exterior como observador extranjero, que también aplicó a sus labores las ideas consideradas avanzadas en su campo.

Francisco Oller Cestero (San Juan, Puerto Rico, 1833-1917), nieto de un renombrado médico homónimo, comenzó su entrenamiento en dibujo y pintura a los doce años ${ }^{3}$. Pronto desarrolló maestría suficiente para recibir encargos eclesiásticos y municipales que le permitieron sufragar estudios en la Academia de Bellas Artes de San Fernando (Madrid), dirigida entonces (18511853) por Federico de Madrazo. Volvió a su patria, pero se asentó en París de 1858 a 1865. Estudió con Thomas Couture, se hizo amigo de Camille Pissarro y Paul Cézanne, y alternó con los ahora conocidísimos Courbet, Monet, Renoir, Sisley, Bazille, y el escritor Emile Zola ${ }^{4}$. Es lógico suponer que se relacionaba también con otros estudiantes puertorriqueños en París por esos años, como Gregorio Tavárez en el Conservatorio y al menos cuatro estudiantes de medicina $^{5}$. Una nueva datación propone 1864 para su obra El estudiante (de medicina), incluida en la colección del Musée d'Orsay'.

Al regresar a Puerto Rico, pintó para iglesias y particulares y envió algunos cuadros a París. En 1868 exhibió 45 obras, abrió una escuela gratuita de dibujo y se casó. Remitió al Salón de París de 1869 la pintura Un boca abajo (el castigo de azotes a un esclavo tendido en tierra), no admitida por "ofensiva a España", pero en 1870 el rey Amadeo lo nombró caballero de la Orden de Carlos III y en 1872, Pintor de la Real Cámara. Por estos años (1867-1871) se sitúa la ilustración de Oller de un informe clínico.

Henri Dumont (París, 1824-Ivry, 1878) estudió abogacía y luego medicina en su ciudad natal (1859) y obtuvo un segundo doctorado en la facultad de medicina de Estrasburgo en 18627 . Allí, en el contexto de la invasión francesa de México (1862-1866), postuló como profesor con una tesis sobre "las enfermedades virulentas y miasmáticas" y publicó, entre otras obras, una Memo-

2 Rigau-Pérez JG, 1985. Stahl, 2015.

3 Delgado Mercado, 1983. Benítez, 1983. Autores varios, 1985. Sullivan, 2014.

${ }^{4}$ La película Cézanne et moi (Danièle Thompson, 2016), sobre la amistad entre el pintor y Zola, incluye el personaje de "Francesco" Oller, sin relevancia en la trama, pero cuyo nombre figura en los créditos: IMDb (Internet Movie Database), https://www.imdb.com/title/tt5078354/reference 19 abril 2018.

5 Menéndez Maysonet, 2007: 18-20. Del Valle Correa, 2003: 172-174.

${ }^{6}$ Musée d'Orsay, París. Índice de artistas: Oller y Cestero, Francisco Manuel: http://www.musee-orsay.fr/en/collections/index-of-works/resultat-collection.html?no_cache=1 19 abril 2018; fechada hacia 1874, que Sullivan, 2014:56, sitúa hacia 1864.

${ }^{7}$ Las biografías de Dumont son breves e incluyen información aproximada o contradictoria. Cito aquí y más abajo la evidencia documental para los datos presentados:

certificado de nacimiento, en los documentos de nombramiento de caballero de la Legión de Honor: Archives Nationales, Paris, base de données Léonore (Légion d'Honneur): http://www.culture.gouv.fr/public/mistral/leonore_fr? ACTION=RETROUVER\&FIELD $1=$ NOM\&VALUE $1=$ DUMONT\&NUMBER $=100 \&$ GRP $=0 \&$ REQ $=\% 28 \% 28 D U$ MONT $\% 29 \% 20 \% 3$ aNOM $\% 20 \% 29 \&$ USRNAME=nobody \&USRPWD=4\%24\%2534P \&SPEC $=9 \& S Y N=1 \& I M L Y=\&$ MAX1=1\&MAX2=1\&MAX3=100\&DOM=All 22 abril 2018;

certificado de defunción, (1979) Boletín de la Academia Puertorriqueña de la Historia 6, 21, San Juan, PR, pp. 150-152, https://archivonacional.com/PL/1/1/12148;

estudios de abogacía, declarados en su informe manuscrito "Sobre las enfermedades epidémicas de las Antillas", 1867/1871, Biblioteca Virtual de la Real Academia Nacional de Medicina (RANM), Madrid http://bibliotecavirtual. ranm.es/ranm/es/consulta/resultados_ocr.cmd?buscar_cabecera=Buscar\&id=11928\&tipoResultados=BIB\&posicion=8 $\&$ forma $=$ ficha 10 enero 2018 ;

títulos de doctor en medicina y cirugía de las facultades médicas de París (25 agosto 1859) y Estrasburgo (30 de agosto 1862) en Arana Soto, 1966:134-140. 
ria sobre los primeros auxilios a los heridos en los campos de batalla ${ }^{8}$. Rápidamente (1863), el ministro de Educación lo envió a México a estudiar la fiebre amarilla y luego pasó a La Habana, de 1864 hasta finales de $1866^{9}$. Investigador y grafómano incansable, sus observaciones sobre los cuerpos y conductas de los esclavos en diferentes haciendas resultaron no solo en informes médicos, sino también en un estudio antropológico (ilustrado por dibujos suyos y fotos) de las etnias africanas en $\mathrm{Cuba}^{10}$. De enero a marzo de 1867 visitó las islas de St. Thomas y Guadalupe y luego se estableció en Puerto Rico ${ }^{11}$. A pesar de que llegó para una época convulsa (en 1868, Grito de Lares en Puerto Rico, Grito de Yara en Cuba, revolución en España; 1870-73, rey Amadeo I; 1873-74, república, restauración borbónica), abrió hospitales de caridad en distintas regiones climáticas de Puerto Rico, para estudiar las enfermedades predominantes ${ }^{12}$. Comunicó sus observaciones sanitarias y ambientales a autoridades médicas y sociedades científicas en San Juan, La Habana, París y Madrid. En Agosto de 1870 fue nombrado caballero de la Legión de Honor.

A poco de llegar a Puerto Rico, estudió las enfermedades, muerte y hallazgos de autopsia de una mujer fallecida súbitamente, cuyo análisis incluyó en varios informes y publicaciones ${ }^{13}$. Los detalles aquí presentados surgen del texto que acompaña la ilustración anatómica de Francisco Oller: un extenso manuscrito (38 caras de papel) enviado a la Real Academia de Medicina de Madrid en $1871^{14}$. Aunque la Academia de Medicina de Madrid, en su sesión de 25 de mayo de 1871 dio cuenta de haber recibido del capitán general de Puerto Rico tres memorias manuscritas por

${ }^{8}$ Baillière et fils, 1864, mencionan las siguientes obras: no. 392. Dumont (H.). Des maladies virulentes et miasmatiques en général; par Henri Dumont, professeur agrégé de la Faculté de médecine de Strasbourg. ln-4, 110 p. Strasbourg, impr. Huder. Faculté de médecine de Strasbourg. Thèse pour l'agrégation; no. 394. Dumont (H.). Mémoire sur les premiers secours à donner aux blessés sur les champs de bataille; conseils aux soldats des armées volontaires. In-8, viii-56 pages. Coulommiers, impr. Moussin.

9 Fredj, 2000. La Académie Impériale de Médecine (París), en sesión del 26 de julio de 1864, recibió los tres informes de Dumont sobre México (topografía médica de Veracruz y observaciones del autor, abril 1863-enero 1864; aclimatación, propagación, incubación y pródromos; relación de síntomas y lesiones anatómicas), referidos por el ministro de Instrucción Pública. La comisión evaluadora de la Academia opinó que "Dumont había comprendido bien su misión y la cumplió digna y concienzudamente", Bulletin de l'Académie Impériale de Médecine 1863-1864; 28:994, 1006-1008. Dumont asiste a sesiones académicas en La Habana desde el 24 de julio de 1864 hasta por lo menos el 25 de noviembre de 1866, según los Anales de la Academia de Ciencias Médicas, Físicas y Naturales de la Habana, 1910-1911, 47:540-541, 926.

${ }_{10}$ Dumont, 2013.

11 "Sobre las enfermedades epidémicas de las Antillas" (ver arriba) se identifica como informe enviado al ministro de Instrucción Pública de Francia, Duruy, desde San Thomas y Guadalupe, enero a marzo de 1867.

12 Arana Soto, 1966:134-140.

13 El caso de Rosa Bey parece estar mencionado brevemente en un extenso y variado informe a la Real Academia de Ciencias Médicas, Físicas y Naturales de la Habana. En su sesión del 11 de diciembre de 1870, acusó recibo de la memoria en francés, "Recherches sur la mégalophthalmos et les maladies des femmes á Puerto-Rico" o "Investigaciones sobre la patología de la muger en las alturas de Puerto-Rico. Del megaloftalmo, del microftalmo y anoftalmo en sus relaciones con el bocio, las afecciones crónicas orgánicas del corazón, de las articulaciones y del útero (cuerpos fibrosos)" y la discutió en cinco sesiones de 1871 y 1872: Anales de la Academia de Ciencias Médicas, Físicas y Naturales de la Habana 1870-71, 7:499; 1871-72, 8:330 (22 de octubre de 1871), 440 (14 de enero de 1872); 1872-73, 9:38 (12 de mayo), 53-54 (23 de junio), 87-88 (14 de julio de 1872).

14 Carta de Gabriel Baldrich, gobernador de Puerto Rico, a la "Academia de Ciencias Médicas de Madrid”, 11 de abril de 1871, Archivo de la RANM, Madrid (ARANM), leg. 117, doc. 5466, con tres informes de Dumont: "Sobre las enfermedades epidémicas de las Antillas" (Signatura: 1-4 A Pasillo / 4-7 , acceso digital, ver arriba); "Recherches sur le megalophthalmos" (Signatura: $1-4^{\circ}$ A Pasillo $/ 4-6^{\circ}$ ), aparentemente, copia de la memoria en francés enviada a Cuba en 1870, y el texto que aquí se comenta, "Buscas [sic — Investigaciones] sobre las enfermedades de las mujeres en Puerto Rico: rotura de la arteria pulmonar a su origen, cuerpos fibrosos de la matris (sic), aneurisma disecante de la aorta, artritis reumática (pierna gambada), exoftalmos y kiste (sic) del ovario; todas afecciones reumáticas". Aparte del quiste del ovario (otra paciente), trata de las enfermedades de Rosa Bey. Lleva fecha de 16 de febrero de 1871, pero la combinación de textos en francés y español, con tachaduras, sugiere cercanía a las notas originales sobre el caso. Incluye la obra de Oller en cara 29. (Signatura: $1-4^{\circ}$ A Pasillo / 4- $8^{\circ}$, http://bibliotecavirtual.ranm.es/ranm/es/estaticos/contenido.cmd?pagina $=$ estaticos/presentacion 10 enero 2018.) 


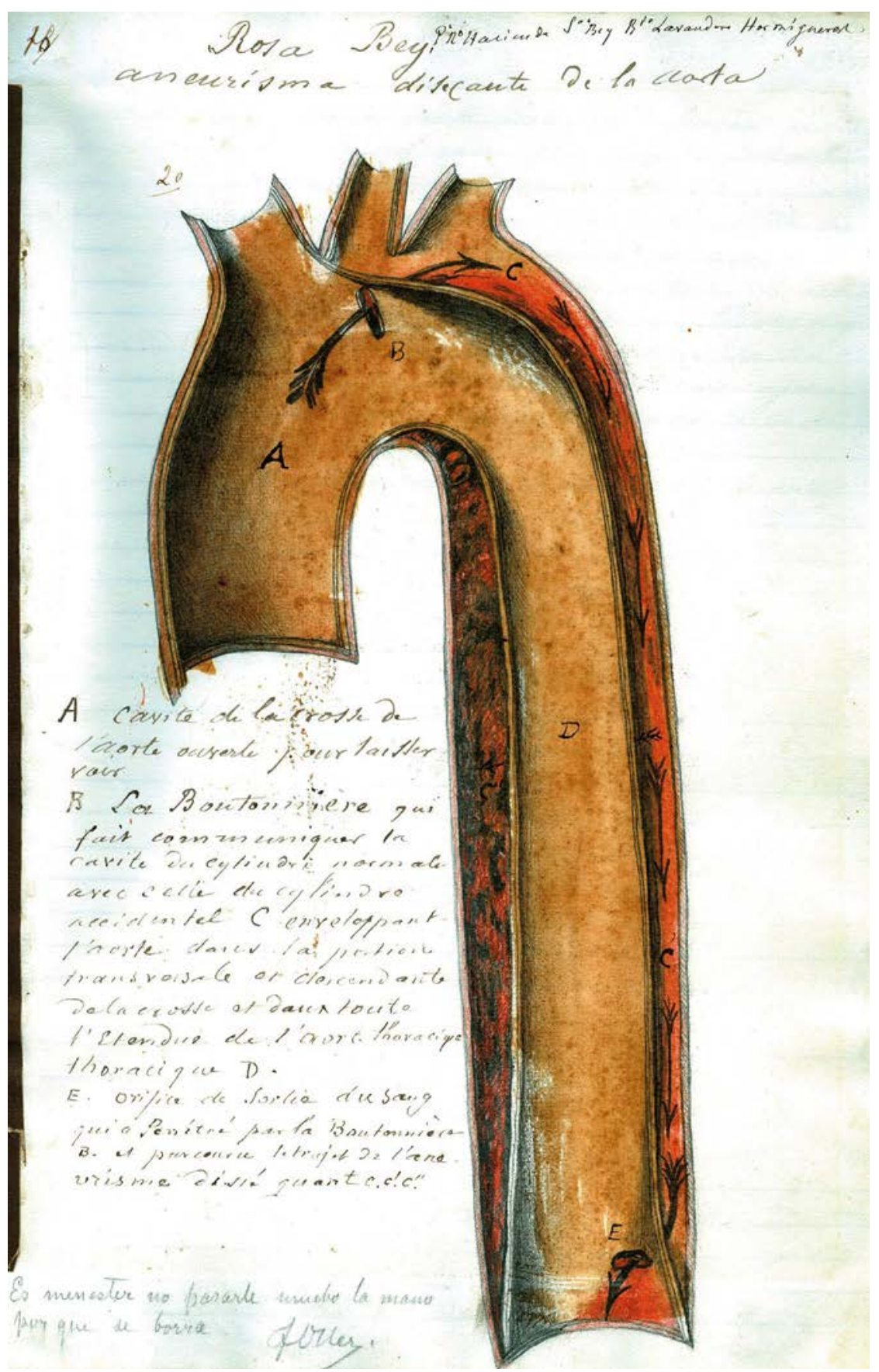

Fig. 1. Francisco Oller, Aneurisma disecante de la aorta, Real Academia Nacional de Medicina, Madrid. Fotografía: Real Academia Nacional de Medicina, Madrid.

Dumont, "que pasaron al examen de las secciones correspondientes", no hay evidencia de una respuesta al médico ${ }^{15}$. Más tarde, Dumont mencionó detalles del caso en su Ensayo de una historia médico-quirúrgica de la Isla de Puerto Rico (dos volúmenes, 1875-1876), que compila sus estudios

15 Libro de Actas de Sesiones Literarias, año de 1870 a 1873, Real Academia de Medicina de Madrid. Acta 25 mayo 1871. ARANM. En nota al margen de la carta de remisión del gobernador Baldrich, citada arriba, dice "En 20 mayo 1871 se acusó el recibo." 
en las divisiones geográficas del país, fundamentados en el examen y tratamiento de cientos de enfermos "ricos únicamente de sus males y de la importancia científica que les acompañaba"16.

El 3 de septiembre de 1867, Dumont participó en una consulta de médicos sobre la extracción de un tumor abdominal a Rosa Bey, "de nación africana, como de 45 años" que llegó a Puerto Rico cuando joven y residía en Hormigueros (oeste de la Isla). El tumor se había manifestado once años antes, junto a menstruaciones exageradas y hemorragias. "La enferma no ha podido más pronto determinarse [a la operación] por oponerse su amo", pero tras la muerte de aquel (por apoplejía) y conseguir su libertad, en cuestión de semanas pidió ser operada. Al examinarla, Dumont palpó "dos tumores grandes pediculados" y "un número indeterminado de tumores sésiles" [pegados al útero]. La auscultación detectó soplos sugerentes de alteraciones de las válvulas cardiacas y las paredes aórticas. El cirujano concluyó que la operación podía ser beneficiosa para evitar los efectos de una hemorragia vaginal severa, pero que el estado del corazón podía constituir una objeción importante. Le informó a la enferma "que las proporciones de las condiciones de muerte son de $90 \%$ por la operación y[,] al contrario[,] que las hemorragias y peritonitis pueden respetar su vida durante meses y años quizás." (Dumont menciona solo complicaciones cardiacas, pero hay que recordar además que, aunque se utilizaba la anestesia general, no se habían adoptado las precauciones antisépticas.)

El 14 de septiembre, "entretanto la enferma se preparaba con las ceremonias religiosas como para determinarse", empezó a gritar: "me ahogo, me ahogo". Dos horas y media después, Dumont la encontró "sentada sobre una hamaca gritando con tanta fuerza que se podían oír los gritos hacia afuera de la hacienda". A pesar de los tratamientos (sangría del brazo, sanguijuelas sobre el corazón, sinapismos o cataplasmas de mostaza, lavativa, purgante y el medicamento cardiaco conocido como digital), Rosa Bey falleció en pocas horas. La minuciosa descripción de la autopsia señaló los ojos saltones ("muy exoftálmicos") de la difunta, fibromas uterinos, y la pierna derecha "gambada" (arqueada) ${ }^{17}$ por deterioro de las estructuras de la rodilla. Reveló, como causa inmediata de la muerte, una ruptura en el origen de la arteria pulmonar, y, además, un aneurisma disecante de la aorta, problema crónico que explicaba los soplos cardiacos detectados en la primera auscultación de la paciente.

El aneurisma es una grieta o apertura en las membranas interiores de un vaso sanguíneo, en este caso la aorta, que lleva la sangre del corazón a los órganos. En la paciente, el aneurisma era disecante: la sangre hizo presión en una fisura de la superficie interior de la aorta y la separó de la capa más musculosa y exterior. Esto muchas veces desgarra la arteria y lleva a muerte rápida por hemorragia. En este caso, la sangre encontró otra fisura en la membrana interior de la aorta para regresar al torrente sanguíneo y produjo una segunda vía (que Dumont llama "cilindro accidental") para la sangre.

En su informe del caso a la Academia de Medicina de Madrid, Dumont quiso mostrar lo que había descrito. Como se ha mencionado, el médico ilustraba gráficamente sus hallazgos por fotografía o sus propios dibujos. Recurrió esta vez a un artista profesional, Francisco Oller. El informe del caso no explica por qué, cómo o cuándo se trazó la imagen. No sabemos si Oller produjo la obra como favor a un amigo (quizás hasta se conocían desde París - pura hipótesis), o, dada la precariedad económica que caracterizó al pintor, por un encargo pagado, que pudo limitarse a la comunicación por correo. Me inclino por esa opción, pues la obra apenas pasa de ser un diagrama. [fig.1] La imagen es casi plana, en contraste con el "hiperrealismo" de los bodegones de plátanos y cocos que pintó Oller décadas más tarde ${ }^{18}$. Además, hay un error en el dibujo. Presenta solo la primera arteria (braquiocefálica) saliendo del cayado de la aorta y otras dos (carótida común izquierda y subclavia izquierda) detrás, como si salieran del "cilindro accidental" creado por el aneurisma disecante. En realidad, las tres arterias salen del mismo tramo de la aorta.

Oller utilizó grafito y lápiz de color, sobre un papel blanco (ahora crema) $(32 \mathrm{~cm}$ x $22 \mathrm{~cm}$ ) cuyo reverso muestra, en filigrana de letras grandes, "J. Jover y Serra". El dibujo se presenta en

\footnotetext{
16 Dumont, 1875-1876: "ricos únicamente de sus males", 1:7-8; Rosa Bey, 1:38-39, 49, 61-85, 90, 92, 2:5.

17 Vaquero/Morales, 2005:354.

18 Sullivan, 2014:137.
} 
fondo blanco, bajo el título "Rosa Bey, Puerto Rico Hacienda de Señor Bey, Barrio Lavandero [sic - Lavadero] Hormigueros; Aneurisma disecante de la aorta". Se muestra la parte ascendente de la aorta (que sale del ventrículo izquierdo del corazón, que no aparece) y las partes transversal (el arco o cayado), descendente y torácica. El interior de la aorta lleva color marrón; el "cilindro accidental" lleva además un sombreado de negro y rojo. Hay letras para marcar detalles importantes y dos flechas que indican la salida de la sangre hacia el "cilindro accidental" y su regreso al interior de la aorta. A un lado está la explicación en francés (entre paréntesis abajo, mi traducción al español):

A Cavité de la crosse de l'aorte ouverte pour laisser voir (Cavidad del cayado de la aorta, abierta para dejar ver)

B La Boutonnière que fait communiquer la cavité du cylindre normale avec celle du cylindre accidentel C, enveloppant l'aorte dans la portion transversale et descendante de la crosse et dans toute l'étendue de l'aorte thoracique thoracique (sic) D.

(El Ojal que hace comunicar la cavidad del cilindro normal con la del cilindro accidental C, envolviendo la aorta en la porción transversal y descendiente del cayado y en toda la extensión de la aorta torácica D.)

E Orifice de sortie du sang qui a pénétré par la Boutonnière B. et parcouru le trajet de l'aneurisme disséquant C. C'. C».

(Orificio de salida de la sangre que penetró por el Ojal B. y discurrió por el aneurisma disecante C. C'. C».)

[En otra letra y a lápiz:] Es menester no pasarle mucho la mano porque se borra F. Oller.

Estas inscripciones sugieren que ambos Oller y Dumont trabajaron en la imagen.

Oller fue un pintor de muchas facetas, en su desempeño (artista y maestro), ideas (liberal, nacionalista, transnacional), influencias (realista, naturalista, impresionista), y obras (paisajes y bodegones, batallas y santos, retratos e interiores) ${ }^{19}$. Gran parte de su creación se ha perdido, pero el monumental cuadro El velorio (1893) provee ejemplo de casi todo eso. En el interior de una morada rústica, veinte adultos, cuatro niños, dos perros y un gato, en acciones diferentes, rodean el cadáver de un infante adornado de flores, tendido sobre el mantel bordeado de encajes en una mesita. De las paredes y las vigas del techo cuelgan objetos y vegetales. En el centro de la composición, un negro anciano, alto pero apoyado en un bastón, contempla el difunto. Al otro lado de la mesa, un joven sostiene un cerdo asado ensartado en una vara. Por dos puertas abiertas se observa el paisaje y la tercera muestra un pedazo de cielo.

¿Qué aporta el descubrimiento de esta ilustración anatómica, pieza mínima en la producción del pintor? Ya se conocían dos tangencias de su vida con la medicina: nieto de médico, amigo y retratista de estudiantes de medicina. La ilustración del caso de Rosa Bey revela su conexión con Dumont y muestra su disposición a poner sus destrezas al servicio de otros profesionales, particularmente, colaborar en un trabajo científico. Edward J. Sullivan, estudioso de Oller, resaltó recientemente su intenso apego "a aspectos concretos de la geografía, luz, flora y costumbres de los habitantes de Puerto Rico". Como Millet y Pissarro, Oller "ambientaba sus representaciones del campesinado puertorriqueño en una atmósfera geográfica y climática, contrastada con un trasfondo sociopolítico"20. También Dumont, en su visión médica, proponía la singularidad de cada región de Puerto Rico (y de cada sitio), pues centraba las enfermedades de sus pacientes en un ambiente específico.

Según la concepción vigente de la historia natural de las enfermedades, la tierra que ocupaba el ser humano, su topografía y su clima, determinaban el estado de salud. En su informe, Dumont presentó una detallada correlación entre los síntomas de la difunta y los hallazgos patológicos,

19 Taylor, 1983:1-7; Sullivan, 2014:2, 4, 6.

20 Sullivan, 2014: 3 ("Oller's intense attachment to the specifics of Puerto Rican geography, light, flora, and the customs of its inhabitants"), 53-54 ("Oller shared Millet's and Pissarro's sympathies for depictions of rural workers as embodiment of the concept of terroir [...] Oller also cast his depictions of Puerto Rican country people in a geographic and climactic (sic — climatic) atmosphere, set against a sociopolitical background [...]". 
uno de los métodos en que se basó el prestigio de la "escuela médica de París" y contribuyó grandemente a comprender las enfermedades ${ }^{21}$. Ante los diferentes problemas de la paciente (ruptura de la arteria pulmonar, aneurisma de la aorta, tumores uterinos, exoftalmos, artritis de una rodilla), Dumont propuso una causa común. Dice: "Una palabra domina la vida y la muerte de Rosa Bey, de toda la raza africana en Puerto Rico, como en todas las Antillas. Esa palabra es REUMATISMO"22. Un diccionario de 1867 valida esa concepción del reumatismo: una diátesis (constitución mórbida que, mediante la alteración de la sangre y los humores, produce enfermedades frecuentes de la misma naturaleza en diferentes puntos del organismo) hereditaria o adquirida, que produce fluxión [hinchazón] aguda o crónica de piel, tejido muscular y fibro-seroso articular o visceral, y que se denomina a veces artritis por su localización habitual en las articulaciones ${ }^{23}$.

Lamentablemente, los datos observados con exactitud por Dumont eran interpretados mediante ideas de causalidad y postulados ambientales y raciales a punto de ser descartados. En pocos años, el descubrimiento de causas específicas, como los gérmenes, produjo el tránsito a una indiferencia a ese ambiente para enfocar en los mecanismos de cambios internos, patología y síntomas. Ya para 1873 otro diccionario define reumatismo como "expresión banal [...] que se aplica a una multitud de dolores que difieren esencialmente en localización y naturaleza" para luego ajustar el término al reumatismo articular como problema específico ${ }^{24}$. También en línea con mentalidades de su época, Dumont no menciona la esclavitud como posible causa accesoria, por sus concomitantes de mala nutrición, trabajo fuerte, poco descanso y escasa atención médica que pueden haber contribuido al desarrollo de los problemas de Rosa Bey.

Dumont falleció el 3 de octubre de 1878, en Ivry, cerca de París. Su muerte se ha atribuido, acorde con su extendida y merecida fama de médico abnegado, a la mordedura de un paciente con hidrofobia (rabia) que le trasmitió la infección. Entonces dejó a su esposa e hija en Yauco, Puerto Rico, y se trasladó a su ciudad natal en busca de cura ${ }^{25}$. Dado que Pasteur no demostró el primer tratamiento exitoso de la rabia hasta 1885, es creíble la tradición familiar de que buscaba remedio a una demencia o pérdida prematura de sus funciones intelectuales ${ }^{26}$.

El año después de colaborar con Dumont, Oller recibió, como se ha dicho, el nombramiento de Pintor de Cámara del rey de España (1872). La Diputación Provincial de Puerto Rico y el Municipio de San Juan sufragaron su viaje a la Exposición Internacional de Viena (1873) para representar la isla con sus pinturas. De regreso, Oller se quedó en París cuatro años y reanudó la compañía de Pissarro y Cézanne. De allí pasó a Madrid en 1877, hasta 1884. En la década siguiente pintó algunas de sus obras más conocidas, como La escuela del maestro Rafael Cordero y El velorio. Viajó a París en 1895 para exponer esta última y pasó en Francia un año, durante el cual Cézanne rompió la amistad que los unía, por un episodio del que no se tienen todos los detalles. Oller regresó definitivamente a Puerto Rico, a pintar y enseñar, hasta su muerte el 17 de mayo de 1917.

\section{Agradecimientos}

Expreso mi agradecimiento a Ignacio Díaz-Delgado Peñas y la Biblioteca-Archivo de la Real Academia Nacional de Medicina, Madrid, España, por su ayuda para llevar a cabo esta investigación. Agradezco también las sugerencias ofrecidas por Carmen M. Santos Corrada, directora,

21 Ackerknecht, 1967: 37-39. Para una reevaluación de estas conclusiones, ver Weisz, 2001.

22 Dumont, "Buscas" [sic - Investigaciones], imagen 42: "Un mot domine la vie et la mort de Rosa Bey, de toute la race africaine á Puerto Rico, comme dans toutes les Antilles. Ce mot c'est RHUMATISME."

23 Bouchut, 1867: 404, 1293.

${ }^{24}$ Littré/Robin, 1873: 1348.

25 Masini/Negroni, Jr./Vivaldi/Mattei, 1925: 116.

26 Rigau Rozas, 2017: 224. 
Biblioteca Conrado F. Asenjo, Recinto de Ciencias Médicas, Universidad de Puerto Rico (UPR); las doctoras Carmen González Keelan y María I. Santé-Pérez, Departamento de Patología, Escuela de Medicina, UPR; y la doctora Mercedes Trelles Hernández, Facultad de Humanidades, UPR-Río Piedras.

\section{BIBLIOGRAFÍA}

Ackerknecht, Erwin H. (1967): Medicine at the Paris Hospital, 1794-1848. Baltimore, Maryland [MD]: Johns Hopkins University Press.

Arana Soto, Salvador (1966): Catálogo de médicos de P.R. de siglos pasados (con muchos de éste). San Juan, PR: s.e.

Autores varios (1985): Simposio internacional "Francisco Oller y su tiempo". En: Horizontes 28, 56 (número especial), Universidad Católica de Puerto Rico, Ponce, PR.

Baillière, J.B. et fils (1864): Bulletin bibliographique des sciences physiques, naturelles et medicales, $4^{m e}$ année, 1863. París: J.B. Baillière et fils. En: <https://books.google.com.pr/books?id=xvgUAAAAQAAJ\&pg=PA50\&lpg=PA50\& $\mathrm{dq}=$ Dumont $+\left(\mathrm{H}_{\mathrm{r}}\right) .+$ Des + maladies + virulentes + et + miasmatiques\&source $=\mathrm{bl} \&$ ots $=\mathrm{GlXzU}$ zqq60\&sig $=1 \mathrm{hRgeWmL}-\mathrm{b}$ c7DHFzzB5qBupGHjs\&hl=en\&sa=X\&ved=0ahUKEwiw-Pb3g6fbAhVBvlMKHb54DgQQ6AEIKzAA\#v= onepage \&q=Dumont $\% 20(\mathrm{H}$.). $\% 20$ Des $\% 20$ maladies\%20virulentes\%20et $\% 20$ miasmatiques $\& \mathrm{f}=$ false $>$ [7 junio 2018].

Benítez, Marimar ed. (1983): Francisco Oller, un realista del impresionismo. [Ponce, PR]: Museo de Arte de Ponce.

Bouchut, Eugène (1867) : Dictionnaire de thérapeutique médicale et chirurgicale. Paris: Germer Baillière. En: $<\mathrm{http} / / /$ www.biusante.parisdescartes.fr/histoire/medica/resultats/index.php?dico $=$ dico \& cote $=27518 \&$ chapitre $=$ rhumatisme $\& \mathrm{p}=1 \&$ do=page $>[7$ junio 2018].

Del Valle Correa, Justino (2003): Los muchachos de París. Trujillo Alto, PR: ed. autor.

Delgado Mercado, Osiris (1983): Francisco Oller y Cestero (1833-1917): Pintor de Puerto Rico. San Juan, PR: Centro de Estudios Superiores de Puerto Rico y el Caribe.

Dumont, Enrique (1875-1876): Ensayo de una historia médico-quirúrgica de la Isla de Puerto Rico, 2 vols. La Habana: La Antilla.

Dumont, Henri (2013): Antropología y patología comparadas de los negros esclavos ( $3^{\text {a }}$ edición en español de la ponencia en francés leída el 13 de mayo de 1876 a la Academia de Ciencias Médicas, Físicas y Naturales de La Habana). San Juan, PR: Ediciones Puerto.

Fredj, Claire (2000): "Cerner une épidémie: le travail des médecins militaires sur la fièvre jaune au Mexique en 1862 et 1867”. En: Genèses, 38, París, pp. 79-104; doi: 10.3406/genes.2000.1609 En: https://www.persee.fr/doc/genes_115532192000 num 3811609 , [ 22 abril 2018].

Littré, Émile/Robin, Charles (1873): Dictionnaire de médecine, de chirurgie, de pharmacie, de l'art vétérinaire et des sciences. $13 e$ édition, entièrement refondue. Paris: J.-B. Baillière. En: <http://www.biusante.parisdescartes.fr/histoire $/ \mathrm{medica} /$ resultats/index.php?dico $=$ dico $\&$ cote $=37020 \mathrm{~d} \&$ chapitre $=$ rhumatisme $\& \mathrm{p}=1 \& \mathrm{do}=$ page $>[7$ junio 2018].

Masini, Juan / Negroni, Jr., Santiago / Vivaldi, J. M. / Mattei, Andrés (eds.) (1925): Historia ilustrada de Yauco. Yauco, PR: Yauco Printing Company.

Menéndez Maysonet, Guillermo (2007): Francisco Pedro Cortés González, músico puertorriqueño de la Belle Époque. Ponce, PR: Casa Paoli, 2007.

Rigau Pérez José G. (1985): "Monstruos, mártires o ejemplos: teratología en Puerto Rico, de 1798 a 1808 ". En: Boletín de la Asociación Médica de Puerto Rico, 77, San Juan, PR, pp. 326-333.

Rigau Rozas, Irma Margarita "Imy" (2017): Una vida, herencia familiar y legado. San Juan, PR: ed. autora.

Sellers, Christopher (2018): "To Place or Not to Place: Toward an Environmental History of Modern Medicine". En: Bulletin of the History of Medicine, 92, Baltimore, MD, pp. 1-45.

Stahl, Agustín (2015): Estudios para la flora de Puerto Rico. 3 vols. Facsímil ilustrado de la primera edición (18831888). Compilado y anotado por Pedro Acevedo-Rodríguez. Washington, DC: Smithsonian Institution.

Sullivan, Edward J. (2014): From San Juan to Paris and back: Francisco Oller and Caribbean art in the era of Impressionism. New Haven, Connecticut: Yale University Press.

Taylor, René (1983): “Introducción”. En: Benítez, 1983:1-7.

Vaquero de Ramírez, María T./Morales, Amparo (2005): Tesoro lexicográfico del español en Puerto Rico. San Juan, PR: Academia Puertorriqueña de la Lengua Española.

Weisz, George (2001): "Reconstructing Paris Medicine". En: Bulletin of the History of Medicine, 75, Baltimore, MD, pp. 105-119.

Fecha de recepción: 13-X-2018

Fecha de aceptación: 13-III-2019 